\title{
Economies criminelles et mondes d'affaire à Tanger
}

criminal economies and the buisiness world in Tangiers

\section{Michel Peraldi}

\section{(2) OpenEdition}

\section{Journals}

\section{Édition électronique}

URL : http://journals.openedition.org/conflits/5973

DOI : $10.4000 /$ conflits. 5973

ISSN : $1777-5345$

Éditeur :

CCLS - Centre d'études sur les conflits lilberté et sécurité, L'Harmattan

\section{Édition imprimée}

Date de publication : 30 décembre 2007

Pagination : 111-125

ISBN : 978-2-296-05120-1

ISSN : 1157-996X

Référence électronique

Michel Peraldi, «Economies criminelles et mondes d'affaire à Tanger », Cultures \& Conflits [En ligne], 68 | hiver 2007, mis en ligne le 10 avril 2008, consulté le 30 mars 2021. URL : http://

journals.openedition.org/conflits/5973; DOI : https://doi.org/10.4000/conflits.5973 


\section{Economies criminelles et mondes d'affaire à Tanger}

\section{Michel PERALDI}

Michel Peraldi est anthropologue, directeur de recherche au CNRS, directeur du Centre Jacques Berque pour le développement des sciences sociales à Rabat (Maroc). Il travaille sur les dynamiques migratoires dans le bassin méditerranéen et les circuits commerciaux informels entre Maghreb et Europe. A récemment publié : Peraldi M., Kokoreff M., Weinberger M. (dirs.), Economies criminelles et mondes urbains, Paris, PUF, 2007.

Cous le terme général d' "économies criminelles », on rassemble usuellement Odes activités qui visent la production, la circulation, la commercialisation de produits interdits d'un point de vue moral ${ }^{1}$ ou légal, des activités dont l'organisation et l'effectuation incorporent une part de violence physique réellement exercée ou potentiellement présente dans l'organisation même du cycle productif, et enfin des activités menées par des individus, des groupes marginaux ou déviants, dans des conditions de totale ou de relative clandestinité. En Italie, où les recherches sur ce thème ont certainement la plus grande ampleur historique, théorique et empirique ${ }^{2}$, les chercheurs ont complété cette définition en spécifiant que ces économies criminelles sont le fait de groupes organisés et hiérarchisés, fondés sur des codes et des rituels d'appartenance (mafia, camorra, n'dranghetta, etc.). Vaille que vaille donc, et surtout à la lumière des travaux italiens ou anglo-saxons ${ }^{3}$ pionniers en la matière, il semblait établi que les économies criminelles concernaient des comportements économiques aberrants, parasites, archaïques, caractéristiques de groupes, d'individus ou de territoires marginalisés, lorsque les défections ou les faiblesses de l'Etat rendaient possible le développement d'une « autorité politique extralégale ». R. Sciarrone précise ainsi que même si ces groupes ont pu s'emparer de domaines économiques et développer leurs affaires

1. Zelliger V., «Repenser le marché : la construction sociale du marché aux bébés aux EtatsUnis ", Actes de la recherche en sciences sociales, $\mathrm{n}^{\circ} 94,1992$.

2. Catanzaro R., Il delitto come impresa. Storia sociale della mafia, Padova, Liviana editrice, 1988 ; Arlacchi P., Mafia et cie, l'éthique mafiosa et l'esprit du capitalisme, Grenoble, PUG, 1986.

3. Ruggiero V., Economie sporche. Limpresa criminale in Europa, Milano, Bollati Boringhieri, 1996; Gambetta D., The Sicilian Mafia: the Business of Private Protection, Cambridge, Harvard University Press, 1993. 
jusqu'à l'échelle mondiale, ils ne changent rien à la force et la nécessité de leur ancrage territorial ${ }^{4}$. Ajoutons enfin une dimension méthodologique essentielle : l'identification de ces acteurs sociaux, individuels ou collectifs passe d'abord par un signalement judiciaire ou policier. C'est en effet d'abord parce que leurs activités tombent sous le coup de la loi et que celles-ci font l'objet de poursuites, que les groupes ou les individus sont « observables » dans des conditions où les chercheurs sont quasi exclusivement tributaires des données policières ou judiciaires. En poussant le raisonnement, on peut alors se demander si le caractère spécifique de leurs activités n'est pas purement déduit, par nature en quelque sorte, du caractère délictueux ou criminel de leurs pratiques. En somme, s'il n'y avait ni meurtres ni violence, pourrait-on parler d'économies criminelles comme d'un registre identifiable, observable de faits économiques ? Parallèlement au débat italien sur la nature économique, institutionnelle et sociale des organisations mafieuses, ravivant la question de la nature «moderne » et capitaliste des entrepreneurs criminels, on assiste aujourd'hui à un double phénomène qui rend nécessaire un retour d'analyse sur l'évolution des phénomènes économiques dit criminels vers la globalisation 5 . D'une part, les organisations criminelles investissent tôt ou tard les économies «légales », et la question se pose alors de l'efficience économique directe des comportements et méthodes mafieux, qui ne peuvent plus alors être ramenés à des mécanismes aberrants ou parasites puisqu'ils sont alors au cœur de l'économie. L'apparition de phénomènes criminels dans les anciens pays du bloc socialiste notamment et surtout le moment de leur apparition ${ }^{6}$, comme immédiate recomposition d'acteurs issus directement des mondes dirigeants de l'ancien régime ${ }^{7}$, pose à notre sens de manière radicalement nouvelle la question des relations entre les acteurs, logiques et dispositifs de l'économie criminelle et ceux des économies « vertueuses ».

En effet, dans la mesure où des logiques et acteurs criminels participent non pas d'un mouvement parasite ou "prédateur » des Etats et des économies capitalistes, mais directement de la (re)naissance du cycle de profitabilité capitaliste, dans la mesure où, pour le dire autrement, des groupes criminels sont les initiateurs, les entrepreneurs schumpetériens dans la renaissance des économies de marché, il est en somme aujourd'hui question de savoir si l'idéal type mafieux, même rendu à la complexité et la sophistication où l'ont amené les chercheurs italiens les plus habiles, peut rendre compte du développement d'économies criminelles à l'intérieur même des mouvements de résurgence ou de formation de cycles capitalistes.

4. Sciarrone R., Mafie vecchie, mafie nuove, radicamente et espansione, Roma, Donzelli, 1998.

5 . Becucci S., Massari M., Globalizzazione e criminalità, Roma, Laterza, 2003.

6. Varese F., The Russian Mafia. Private Protection in a New Market Economy, Oxford, Oxford University Press, Paperback, 2001, 2005 ; Favarel-Garrigues G., « Concurrence et confusion des discours sur le crime organisé en Russie », Cultures E Conflits, n²42, 2001.

7. Volkov V., Violent Entrepreneurs. The Use of Force in the Making of Russian Capitalism, London, Cornell University Press, 2002. 
Rendue aux dimensions d'une anthropologie urbaine - car cette renaissance prend des formes très diffuses en milieu métropolitain - ce développement pose d'une tout autre manière le fait que, d'autre part, on assiste à l'extension et à la diffusion des économies criminelles bien au-delà des lieux historiques de l'émergence de phénomènes mafieux historiques (Espagne, pays de l'ancien bloc socialiste) et ces phénomènes d'émergence invalident l'hypothèse de l'ancrage territorial comme matrice sociale explicative des phénomènes.

Particulièrement sensible en milieu métropolitain, ce développement pose d'une tout autre manière que par le passé, la question de la nature et des modalités d'organisation de ces économies criminelles dans la ville. Il semble en effet désormais difficile de raisonner comme si ces économies relevaient d'une « niche » sociale autonome, hors de l'espace-temps de la ville dont elles occupent parfois une grande partie de la population... On constate que les sciences sociales hésitent entre la tentation de sous-estimer l'impact de ces économies dans les mondes urbains en les limitant à certains mondes ethniques ou sociaux, avec le risque d'une ethnicisation criminalisante des mondes impliqués 8 ou, au contraire, la surestimation paranoïaque du contrôle et de la puissance des entrepreneurs et organisations de l'économie criminelle 9. Comment prendre la mesure réelle de la place qu'occupent les économies criminelles dans les économies urbaines, et surtout comment définir la consistance propre des mondes qu'elles occupent, leur place et statut dans l'univers métropolitain ? La question est d'importance, mais ne peut être traitée que si justement on rend compte des porosités comme des fractures entre mondes économiques dans la ville.

C'est cette question qui nous guidera dans la présentation d'une campagne de recherche que nous commençons ${ }^{10}$ sur les activités économiques émergentes dans l'une des régions urbaines du Nord du Maroc, la métropole Tanger Tétouan.

\section{La ville blanche....}

Tanger est une de ces villes périphériques auxquelles est attachée une réputation criminelle. Ici, comme à Marseille, Naples ou Chicago, il semble que cette réputation se soit fabriquée dans les années 1930, à la faveur de films de série $\mathrm{B}$ ou de romans noirs évoquant la ville et sa «pègre » en un subtil mélange d'exaltation romantique orientaliste et de désignation stigmatisante.

8. Bourgois P., En quête de respect, Paris, le Seuil, 2001 ; Bouhnik P., Le Monde social des usagers de drogue dures en milieu urbain défavorisé, thèse de doctorat, université Paris-8, 1995.

9. Saviano R., Gomorra, Milano, Ed. Mondadori, 2006.

10. Cette recherche, entamée il y a un an, porte sur les mondes d'affaire à Tanger, à partir des méthodes classiques de l'anthropologie, récits de vie, observation participante et monographie d'entreprises. Nous nous sommes pour l'instant limités à la description d'un groupe informel d'investisseurs et nous avons entrepris un recueil systématique d'articles de presse sur les affaires criminelles de la région, notamment sur le trafic de cannabis. Enfin, nous commençons un travail monographique sur les entreprises du secteur de la confection à Tanger. 
Tandis que la France et l'Espagne se partagent le Maroc, sous statut de protectorat espagnol dans la frange côtière nord, et français pour l'immense reste du pays, dès 1925 et jusqu'en 1960, Tanger fut placée sous statut de ville internationale. Elle est alors gérée par un Comité de contrôle dont les grandes nations européennes - Espagne, France, Italie, Allemagne et Angleterre - sont membres de droit ; les autorités françaises et espagnoles y ont mandat seulement pour la gestion administrative de la ville. Ce statut très spécial y organise un " cosmopolitisme politique » de type alexandrin ${ }^{11}$, autant qu'il permet, par le caractère de port franc accordé à la ville, le développement d'un affairisme déjà à dimension internationale. Tanger sera, même après l'indépendance du Maroc, un lieu de refuge et d'activité pour des mondes d'affaires aux limites du banditisme, autant qu'une place marchande et de transit pour des activités franchement criminelles : prostitution, jeux clandestins, drogue, contrebande dans lesquelles sont impliquées quelques grandes figures du banditisme corse ${ }^{12}$. Lucky Luciano, grand patron de la mafia new-yorkaise y aurait eu lui aussi des affaires. Cette période, même révolue, fait mémoire, « capital social » transnational.

La ville tombe en léthargie dès son entrée dans le Maroc indépendant, tenue en ostracisme par le pouvoir royal. Dans les années 1960-1970, la ville retrouve une aura internationale en figurant dans les circuits et lieux d'initiation de la Beat Generation américaine, attirée là par quelques écrivains pionniers (Truman Capote, Paul Bowles, William Burroughs, Jack Kerouac) qui y résident plus ou moins longuement, parfois hébergés par une bourgeoisie américaine installée dans la période internationale, attirés aussi par le cannabis cultivé dans les montagnes du Rif, à une centaine de kilomètres de la ville. Chefchaouen ou Kettama ont été, dans les années 1960, des lieux de pèlerinage pour la Beat Generation, à l'égal d'un Katmandou ${ }^{13}$.

Cette culture va rapidement se développer dans les années 1970-1980, bénéficiant d'une part de l'engouement du marché européen pour ce produit, d'autre part de l'arrêt des cultures dans les pays classiquement producteurs - Afghanistan et Liban- qui basculent dans l'état de guerre. Aujourd'hui cette culture occupe de manière quasi absolue les terres cultivables de la région montagneuse comprise entre Chefchaouen à l'Est et et Keitama à l'Ouest ${ }^{14}$. Selon un rapport établi à la demande de l'OFDT, le Maroc occupe la place de premier producteur mondial de cannabis, fournissant $80 \%$ des produits dérivés consommés en Europe. Ce même

11. Ilbert R., Alexandrie, histoire d'une communauté citadine, Le Caire, IFAO, 1992.

12 . Follorou J., Nouzille V., Les Parrains corses, leur histoire, leurs réseaux, leurs protections, Paris, Fayard, 2004.

13. Moreno P., Estudio del cultivo de cannabis sativa en el Rif marroqui: sus consecuencias socioeconomicas para la region, Universidad politecnica de Valencia, Ecuela Tecnica Duperior de Ingeniores Agronomes, Depto de Economia y Ciencia Sociales, décembre 1997.

14. Selon l'ADPN, organisme marocain de développement pour la région nord (Enquête sur le cannabis, 2003), les surfaces cultivées sont passées de 35000 hectares en 1975 à 134000 aujourd'hui, sur une zone qui s'étend d'Al Hoceima à l'Ouest, à Larache à l'Est de la région. 
rapport rédigé en 2001 estime à 12 milliards d'euros les bénéfices de cette agriculture, dont une très faible partie revient aux agriculteurs ${ }^{15}$.

On reviendra sur les formes et l'organisation transnationale de cette économie du cannabis. Gardons pour l'instant à l'esprit que, dans les années 1980, le cannabis est certainement la première source de devises de cette région nord du Maroc. Difficilement mesurable, son impact dans Tanger est pourtant visible puisque, dès la fin des années 1970, on voit s'édifier des centaines d'immeubles, de villas parfois très luxueuses sans qu'aucune prospérité urbaine ne puisse expliquer ni ce développement ni la fièvre immobilière qui l'accompagne. Certes, le port franc et la perspective d'acheminer sous douane un certain nombre de produits suscitent l'installation d'industriels, certains chassés de Casablanca, la capitale, par ses encombrements et son développement anarchique. C'est le cas notamment de la filiale marocaine d'un grand fabricant français de produits de céramique pour salles de bain, leader mondial, dont l'installation va devenir le chef de file de la création d'une première zone industrielle à Tanger. Le développement industriel aujourd'hui manifeste de la ville ne démarre réellement qu'à la fin des années 1980, avec la création de zones franches industrielles où s'installent des entreprises européennes qui y délocalisent tout ou partie de leur production. C'est entre autres le cas d'entreprises du secteur textile, parmi lesquelles de nombreuses entreprises espagnoles, dont le leader du marché, Zara, l'un des principaux donneurs d'ordre pour les ateliers tangérois.

Dès la mort du roi Hassan II, son fils, Mohammed VI, qui lui succède, décide du lancement d'un grand programme de rénovation et d'aménagement à l'échelle de cette région urbaine ${ }^{16}$. Le fer de lance de ce programme est l'ouverture d'un port franc nouveau à 60 kilomètres de Tanger, programmé pour accueillir les plus grands navires porte-containers et drainer une partie du trafic maritime euro-méditerranéen. L'Etat marocain assure la moitié des financements du port et de ses infrastructures directes, le reste est assuré par des capitaux privés en partie étrangers (notamment issus des Emirats arabes unis, Koweït et de Dubai). L'Etat assure en totalité la construction des dessertes ferroviaires et autoroutières du nouveau port. Une première tranche a été inaugu-

15. On y produit environ 48000 tonnes de plantes et 3000 tonnes de résine, qui nourrissent environ 100000 exploitants agricoles lesquels bénéficieraient de 20 millions d'euros sur les 10 milliards que génère le trafic (voir Labrousse A., Romero L., Maroc, La Production de cannabis dans le Rif, Observatoire français des drogues et toxicomanies, bulletin mensuel, $\mathrm{n}^{\circ} 13$, février 2002).

16. Les raisons de cet engouement soudain pour Tanger sont difficiles à identifier, car rarement exprimées. Il semble que le nouveau roi ait eu très rapidement la volonté de corriger, y compris par des gestes hautement symboliques, les erreurs et surtout les ostracismes du régime précédent. La région nord, littéralement abandonné par l'Etat, va bénéficier ainsi au titre d'une réparation autant économique que morale, d'un investissement exceptionnel. Cela n'empêche par pour autant - on y reviendra - que des conjonctions d'intérêts dont l'Etat était porteur puissent aussi se manifester en faveur d'un développement vers le Nord, y compris une volonté d'arrimage économique à l'Europe proche. 
rée en juillet 2007, l'achèvement des travaux étant prévu pour 2010 17. L'Etat est aussi partie prenante d'une série d'initiatives d'aménagement prises en faveur de toute la zone littorale dont le développement touristique est organisé en partenariat avec de grands groupes immobiliers, espagnols surtout, mais aussi koweitiens, émiratis ou anglais. Pour montrer, s'il le fallait, sa détermination, le roi a nommé à Tanger un walli 18 réputé "à poigne », auréolé de la gloire qui lui revient dans le développement et la prospérité de Marrakech dont il a orchestré la renaissance tout au long des années 1980. Fait hautement symbolique, les riches et célèbres européens, écrivains, artistes, couturiers et acteurs de cinéma qui ont activement contribué à la gentrification de la médina de Marrakech achètent et rénovent des bâtisses aujourd'hui dans la kasbah de Tanger.

\section{Une zone métropolitaine striée de frontières}

Partant de l'Atlantique et sur un front méditerranéen d'une centaine de kilomètres, s'étend donc une région urbaine discontinue, ponctuée même de ruralités profondes telles les deux montagnes du Rif. Plus à l'Ouest, Tanger, aujourd'hui ville d'un million d'habitants, constitue le second pôle industriel du pays, après Casablanca grâce notamment à ses zones franches et zones industrielles où se sont délocalisées des entreprises européennes. Tanger est en revanche, même avant la construction de Tangermed, le premier pôle portuaire du pays pour le trafic de passagers et qui s'apprête donc, avec sa nouvelle zone portuaire, à devenir le premier port industriel du pays et sans doute l'un des tout premiers de la Méditerranée. Ce nouveau pôle portuaire tisse encore plus de continuité entre Tanger et le second pôle urbain de cette région, Tétouan, ville administrative de 700000 habitants un peu en retrait dans les terres. Elle était une belle endormie jusqu'à très récemment, seulement réveillée par le dynamisme de son front maritime ouvert à l'urbanisation touristique dans les années 1980. La troisième pointe du vague triangle que forme ce dispositif urbain est constituée par une ville très particulière qui occupe la presqu'île de Sebta, (Ceuta en espagnol), enclave espagnole en terre marocaine ${ }^{19}$. Depuis le renforcement des contrôles et la pression de candidats subsahariens à la migration vers l'Europe, Sebta s'est séparée de ses voisins marocains par un dispositif frontalier très important; un dispositif à l'israélienne, formé d'un complexe de patrouilles militaires, de mur et

17. Le Tellier J., Les Recompositions territoriales dans le Maroc du Nord, thèse de doctorat de géographie, université Aix-Marseille 1, octobre 2006.

18. Equivalent d'un préfet, à ceci près qu'il est nommé par le roi auquel il réfère directement.

19. Le Maroc, à la fin du protectorat, maintien deux enclaves en terre marocaine : Ceuta, entre Tanger et Tétouan, et Melilla, plus à l'Est, à proximité du port de Nador. Les raisons géopolitiques de ce maintien ne sont pas claires. Attachement culturel à des zones qui ont joué une rôle majeur dans l'histoire espagnole de ce siècle (l'armée espagnole fasciste de Franco se constitue à Ceuta et, de là, part à l'assaut de l'Espagne républicaine), réponse cathartique à l'occupation anglaise de Gibraltar, juste en face, sans doute. Reste qu'aujourd'hui, les deux entités espagnoles sont des lieux commerciaux très stratégiques d'où s'organise un commerce frontalier intense avec le voisin marocain. Sur l'histoire de l'enclave, voir Driessen $\mathrm{H}$, On the Spanish-Moroccan Frontier, A Study on Ritual, Power and Ethnicity, Oxford, Berd, 1992. 
de barbelés. Mais cette clôture n'empêche pas l'intensité des liaisons et l'instauration d'une continuité urbaine sans cesse plus visible, à la mexicaine. La ville marocaine mitoyenne de Fnidek est aujourd'hui une extension économique de Sebta ; le souk de la ville n'a quasiment plus d'autre fonction que de commercialiser les produits sortis des supermarchés de la voisine espagnole. En outre, l'espace se raréfie à Sebta, coincée sur sa presqu'île, et des citoyens espagnols font mobilité résidentielle vers le Maroc en nombre sans cesse croissant. C’est donc un espace de continuités urbaines qui se fabrique là progressivement, selon le principe connu de l'extension/débordement des périphéries les unes sur les autres (Tanger s'étend vers Sebta et Tétouan, qui s'étendent vers Tanger). Mais c'est aussi un espace de discontinuités profondes, une entité métropolitaine striée de frontières culturelles - entre Tanger l'internationale et Tétouan la traditionaliste - politique voire « impériale » entre Sebta et ses voisines, sociales enfin car les contrastes sociaux sont marqués entre l'intérieur des villes, les anciennes médinas lieux d'un habitat toujours populaires, les immenses périphéries bidonvillisées de Tanger et Tétouan, que l'on dit aujourd'hui gagnées à l'islamisme, et les fronts maritimes, de plus en plus « marbellisées 20 », et gagnés à une clientèle touristique encore en grande partie composée de migrants marocains désormais coupés de leur base familiale et qui cultivent là un hédonisme exacerbé. Adossées à cette zone urbaine maritime, les montagnes sauvages du Rif, dévolues aujourd'hui pour l'essentiel à la culture du cannabis.

\section{Une métropole transnationale?}

Cette métropole ne forme pas davantage un ensemble économique cohérent et hiérarchisé ni surtout sans zones d'ombres. Outre l'économie du cannabis, cette région a déployé de longue date des réseaux de contrebande. Les habitants de Tétouan bénéficient d'un droit d'entrée de 24 heures à Sebta, ils assurent par ce biais, depuis des décennies, un commerce florissant en liaison avec les principaux marchés et souks urbains et ruraux de la région. Les marchandises en provenance de Sebta, vêtements, parfums, cosmétiques, produits alimentaires irradient via les «professionnels » de la région, tous les centres urbains du Grand Nord marocain, jusqu'à Casablanca ${ }^{21}$. A Tanger même, les réseaux de contrebande sont très actifs depuis longtemps, à partir de l'Espagne elle-même, que seuls quatorze kilomètres séparent de Tanger, et aujourd'hui avec le renforcement de la surveillance policière sur le détroit, depuis les zones franches implantées dans la ville elle-même. L'une de ces trois zones est ainsi installée depuis les

20. Du nom de la ville de Marbella, célèbre station balnéaire de la côte espagnole, en face, et dont l'urbanisme touristique expansif sert aujourd'hui de modèle au développement de l'urbanisme touristique au Maroc.

21. Ceuta, enclave espagnole de près de 80000 habitants incluant une minorité musulmane, est une place marchande de produits dédiés à la contrebande. 10000 personnes, dont les habitants voisins de Tétouan, entrent sans visa chaque jour à Ceuta, dont l'activité commerciale déborde largement sur les communes marocaines voisines (Martil, Fnideq), mais aussi Tanger, Tétouan, pour un revenu d'activité estimé à 2 milliards d'euros. 
années 1970 sur le port, en plein centre-ville. Fermée de murs d'enceinte comme une forteresse, son étanchéité constitue très largement une illusion. Des tonnes de marchandises sont envoyées la nuit depuis les toits terrasses des entrepôts et des ateliers sur la plage qui jouxte la zone et gagnent les marchés et les souks de la région urbaine. L'installation et le développement d'un important tissu d'entreprises de confection délocalisées ${ }^{22}$ génère aujourd'hui deux nouvelles formes de contrebande : d'une part, avec la complicité des ouvrières et de certains patrons d'atelier, la sortie clandestine de marchandises déclarées déclassées ou faillies. Des modèles de grandes marques espagnoles, allemandes ou françaises se retrouvent ainsi dans les boutiques du centre-ville de Tanger ou se vendent « par relation » dans les cafés de la ville. D’autre part, on trouve la contrefaçon, qui peut prendre toutes les formes possibles, de l'imitation grossière et sommaire de modèles de grandes marques à la très impeccable copie de ces mêmes modèles, réalisées dans les mêmes tissus et sur les mêmes maquettes, souvent fournis aux ateliers clandestins par les patrons ou les ouvriers des usines des zones franches. Tous ces commerces, même s'ils alimentent d'abord le marché local marocain, y compris celui des grandes villes, sont dynamisés par la très forte présence touristique et transitaire, à Tanger même pour le transit, sur toute la côte urbanisée pour le tourisme ${ }^{23}$. Le tourisme, autre industrie locale en plein développement, est aujourd'hui d'abord une industrie immobilière. On vise un tourisme de résidence plus que de passage, et la côte se couvre inexorablement de lotissements pavillonnaires déserts l'hiver. Un chapelet de cités balnéaires s'étend donc désormais sur tout le littoral tétouanais, depuis Sebta jusqu'à Cabo Negro, la plus courue des stations balnéaires de la zone. On attend pour les deux prochaines années l'investissement de grands groupes de tourisme immobilier espagnols et émiratis (Dubaï) à l'Ouest (Asilah, Larache) et à l'Est, sur la nouvelle zone portuaire. Cette frénésie immobilière qui n’est pas sans rappeler celle qui a gagné les côtes espagnoles ces trente dernières années, embraye sur la frénésie constructive strictement urbaine que connaît Tanger depuis le développement du commerce de cannabis ${ }^{24}$. Des quelques données qui ressortent des plus récentes affaires, il semble bien que l'immobilier soit là aussi le secteur privilégié dans lequel viennent investir, tant les paysans enrichis par le cannabis que les commerçants et passeurs espagnols et marocains.

C'est le seul point commun de l'ensemble des économies de cette zone que de s'appuyer sur le différentiel frontalier comme ressource : les délocali-

22. On compte aujourd'hui 278 entreprises qui travaillent à Tanger dans la filière textile, $80 \%$ d'entre elles travaillent à l'exportation dans les trois zones franches industrielles que compte désormais la ville.

23. Selon les années, le trafic maritime de passagers sur les ports de Tanger et Sebta, varie de 3,5 millions de passagers à 4 millions. Il est en constante progression et très saisonnier, concentré sur les quatre mois de l'été.

24. Selon la presse locale (Les Nouvelles $d u$ Nord), les prix de l'immobilier augmentent de $30 \%$ par an depuis le début des années 2000. Renseignements pris dans les agences tangéroises, un appartement neuf de semi-standing sur le front de mer se négocie à 2000 euros le $\mathrm{m}^{2}$. Le même appartement coûtait 1200 euros le $\mathrm{m}^{2}$ au début des années 2000. 
sations industrielles, la contrebande, le tourisme, et même l'activité portuaire jouent à leur manière du différentiel frontalier. L'économie urbaine est une économie frontalière, elle est aussi une économie largement dominée par des acteurs transnationaux, ne serait-ce que par les relations avec l'enclave espagnole de Sebta. Mais en réalité, ce transnationalisme franchit allègrement le détroit. Une partie des entreprises délocalisées ou filialisées des zones industrielles et des zones franches sont des entreprises à capitaux mixtes, marocoespagnols ou maroco-français.

On retrouve ces mêmes caractéristiques dominantes - transnationalisme et commerce frontalier - dans le trafic de cannabis.

\section{Le cannabis, une économie segmentée et socialement diffuse}

Il est bien clair qu'il s'agit d'une activité et d'organisations sur lesquelles on manque cruellement de données empiriques anthropologiques. Très peu de travaux ont été menés au Maroc ou tout au long des routes commerciales du cannabis ${ }^{25}$. De même, on manque de données judiciaires ou policières, tant au Maroc que dans les pays d'Europe concernés ${ }^{26}$.

Certes, les chiffres globaux sont connus, on l'a vu. Le Maroc produit donc environ 3000 tonnes de résine de cannabis par an fournissant ainsi bon an mal an, près de $80 \%$ du marché européen. Le produit est cultivé dans des montagnes escarpées, difficiles d'accès, mais surtout dans une région où les alternatives sont difficiles à mettre en place, il y a encore peu, misérable et frondeuse ${ }^{27}$. Une partie des services publics locaux est complice et contribue à la circulation du produit, l'Etat tolère faute d'un véritable projet alternatif. Le produit est transformé sur place, de la fleur à la résine ${ }^{28}$. Mais on trouve aussi des ateliers de transformation éparpillés dans toute la région urbaine, Tanger y compris. Les consommateurs

25. Voir Moreno P., op. cit.

26. Que ce soit l'occasion de dire ici que l'accessibilité donnée par les Commissions italiennes (antimafia et anti-camorra) sont des exceptions dans l'ordinaire des rapports entre chercheurs et institutions policières et judiciaires. Certes, on peut recueillir des chiffres globaux, élaborés par les institutions elles-mêmes, on peut s'entretenir avec des acteurs confrontés au trafic, qui sont d'ailleurs parfois de très bons connaisseurs des circuits et des filières. Mais l'accès direct aux données de base (procès verbaux, minutes des procès) est strictement impossible en France, très contingenté en Espagne. Or, seules ces données directes permettent de construire des régularités sociologiques et historiques. Au Maroc pour l'instant, les affaires traitées par la justice sont trop peu nombreuses pour qu'il soit possible encore d'envisager de compiler des dossiers.

27 . Berriane M., Signoles P., Les Espaces périphériques au Maroc et au Maghreb à l'heure de la mondialisation, Rabat, université Mohammed V, 2000.

28. Il faut 30 kilos de fleurs pour faire un kilo de résine, la fleur est payée 2,3 euros, la résine est payée 300-400 euros le kilo selon la qualité au départ du Maroc et atteint entre 1500 et 2000 euros au détail sur les marchés européens. On estime que ce trafic a généré près de 11 milliards d'euros de recettes. Un quart de la surface agricole du Rif est dévolu à cette culture. On y produit environ 48000 tonnes de plantes et 3000 tonnes de résine, qui nourrissent environ 100000 exploitants agricoles lesquels bénéficieraient de 20 millions d'euros sur les 10 milliards que génère le trafic. 
affirment que plus le produit est fabriqué loin de sa base rurale, plus il est frelaté. L'essentiel du produit passe donc en Espagne ${ }^{29}$, bien qu'il soit possible d'identifier des filières directes vers la France via l'Algérie. De nombreuses opérations de saisie dans les ports de Casablanca, Agadir, et même Dakar, sur des bateaux en partance pour l'Europe, témoignent également de la diversification des lieux et modes d'embarquement, et partant, de l'extension et de la démultiplication des dispositifs d'acteurs impliqués dans l'économie du cannabis. A en juger par les quelques saisies effectuées ces dernières années sur le port de Tanger principalement, le mode de passage le plus usité semble celui de chargements dissimulés dans des camions, notamment frigorifiques, dont le transit par Tanger est en grande augmentation, notamment depuis le développement de la filière des fruits et légumes (le Maroc exporte des tomates notamment vers l'Europe). Mais d'importantes saisies ont aussi été opérées dans des camions transportant vers l'Europe des produits de confection élaborés dans les usines sous douanes des zones franches 30 .

Ce mode de passage le plus usité s'encastre donc totalement dans la logistique du dispositif industriel transnational euro-marocain. On manque de données d'enquête, mais il semble a priori difficile de penser que cet encastrement puisse se faire sans que les entrepreneurs, transporteurs, fabricants soient au courant, même si, au vu des interpellations et inculpations, on peut se rendre compte que les chauffeurs routiers ont bon dos...

Le second mode de passage, de loin le plus médiatisé ${ }^{31}$, empruntant les routes et les moyens parfois spectaculaires de la contrebande, dans un jeu codé avec les polices douanières marocaines et espagnoles, relève d'une échelle artisanale. Des bateaux très rapides partent donc la nuit des multiples criques et plages de la côte tangéroise pour rejoindre les mêmes criques cachées côté espagnol. C'est un mode de passage qui n'est pas sans rappeler les techniques qu'utilisaient les contrebandiers de cigarettes à Naples jusque dans les années 1980. C'est ce mode de passage qui fait aujourd'hui l'objet du plus grand nombre d'affaires portées devant la justice. L'affaire Bin Louidane en est un exemple parmi d'autres. Durant l'été 2006, la police marocaine arrête celui qui va devenir, dans toute la presse locale et au-delà, le principal protagoniste de l'affaire Bin Louidane. C'est là en fait le surnom, ou mieux le « nom

29. L'Espagne est au premier rang mondial des saisies (50\% du total des saisies européennes), pas seulement parce qu'elle est le débouché naturel le plus proche du Maroc, mais parce qu'elle est aussi un lieu d'organisation du commerce. En 2005 par exemple, à Marbella, un vaste réseau a été démantelé par la Guardia Civil, à la tête duquel figurait un avocat chilien et diverses personnes de milieux politiques espagnols, financiers, 62 millions d'euros saisis et des centaines de sociétés fictives, surtout immobilières, mises à jour (Le Monde, 17 mai 2006).

30. Pour la seule année 2005, 10 tonnes de produit ont été saisies, la quasi-totalité à bord de TIR (Transports routiers internationaux), et 247 personnes arrêtées, dont une centaine d'Européens (en majorité des Espagnols et des Français). Le record des saisies concerne un camion de tomates en provenance du Sud marocain qui transportait pas moins de 5 tonnes de résine de cannabis.

31. Cette contrebande a même donné lieu à un roman à succès, par ailleurs très bien informé sur les modes de passage et de circulation : Reverte A. P., La Reine des sud, Paris, Gallimard, coll. « folio », 1998. 
de guerre ", que s'est choisi l'homme arrêté et que l'on considère comme l'un des principaux trafiquants de cannabis du Maroc. Fils d'un paysan pauvre du Rif, Mohamed El Kharraz de son vrai nom, débute dans le métier comme modeste muletier dès les années 1980 puis, construisant un réseau d'affaire entre Espagne et Maroc, il apparait à la fin des années 1990, après un premier passage en prison, comme l'un des plus puissants trafiquant de la région.

Mais la presse ne s'intéresse pas à l'affaire et ne lui donne une grande audience que pour ses implications politiques. Bin Louidane donne en effet son carnet d'adresses aux gendarmes qui l'arrêtent ; un carnet où figure une longue liste de notables influents, dont un ancien commissaire divisionnaire de Tanger devenu chef de la sécurité personnelle du roi et pas moins d'une vingtaine de dignitaires, magistrats ou officiers des douanes, de la police régionale. Tous font aujourd'hui l'objet de poursuites. Pour achever le portrait médiatique du personnage, on lui prête une sympathie pour les groupes islamistes, un journal local fait même état d'entrevues régulières entre Bin Louidane et le chef d'un important mouvement islamiste radical en vacances dans la région. L'importance médiatique de l'affaire donne à la presse une raison d'abandonner le terrain des approximations, au profit de descriptions minutieuses, presque cliniques de l'affaire. Les minutes du dossier judiciaire paraissent d'ailleurs en feuilleton dans un hebdomadaire marocain ${ }^{32}$.

Bin Louidane possède une flotte de puissants bateaux avec lesquels il travers la vingtaine de kilomètres du détroit de Gibraltar pour acheminer le produit en Espagne. Ses chargements dépassent rarement quelques centaines de kilos, ce qui donne, par-delà le folklore, une dimension artisanale à son commerce. On l'a vu, les camions frigorifiques et autres acheminent le produit par tonnes. Sur le plan de son organisation, le dispositif mis en place semble bien plus réticulaire et familial que pyramidal et "organisé », au sens clairement mafieux du terme. Impliquant son frère, ses beaux-frères, beau-père, épouse entre autres membre de sa famille, le « clan » Bin Louidane ressemble plus à une petite entreprise familiale qu'à une organisation mafieuse et ce, d'autant plus qu'elle fonctionne par «coups » de transports dont les protagonistes sont rarement les mêmes d'une campagne à l'autre. Chaque montage de coup peut en effet s'effectuer avec des investisseurs, des agriculteurs fournisseurs, des clients et des «marins » différents. La seule compétence du « savoir passer » mobilise l'entreprise.

En un mot, Bin Louidane semble plus un moderne monteur d'opération, jouant pragmatiquement d'opportunité, qu'un chef d'organisation. Qu'il cherche à accumuler de la puissance et de l'influence autant que des richesses, ce qui l'a sans doute perdu, tient moins à une efficience économique qu'à la

32. Il s'agit de la Gazette $d u$ Maroc, mais l'affaire est aussi largement commentée dans les autres hebdomadaires marocains (Le Journal, Tel Quel). Effet transnational également, il est fait mention de cette affaire dans des quotidiens espagnols. 
conquête d'un statut dans une région encore fortement marquée par les traditions rurales cherifiennes. Ben Louidane, contrairement justement à la comparaison facile, apparaît comme un « monteur ", un opérateur qui met en relation parfois de vastes réseaux d'acteurs, sans pour autant que ces acteurs, occasionnels et réguliers, ne constituent les membres d'une « organisation».

Le « dispositif » Bin Louidane, se déploie dans un territoire très fortement transnationalisé, entre la région côtière de Tetouan, Sebta et l'Espagne, Marbella surtout. Car, si le produit est bien cultivé dans le Rif, préparé dans la région de Tetouan, les financements, les passeurs, la logistique technique sont ancrés d'une part sur la côte espagnole face au Maroc - Marbella, Algesiras et même jusqu'à Cadix, et surtout dans la presqu'île de Sebta. Cette structure transnationale se retrouve au niveau des investissements et des placements financier $\mathrm{du}$ réseau : son patrimoine se compose de fermes agricoles très modernes au Sud de Tanger, où il fait élever des chevaux, une villa, des immeubles de bureaux, une usine de confection, des terrains à bâtir à Tanger, une villa à Tetouan, des terrains et un appartement à Marrakech, des magasins dans un centre commercial très chic à Casablanca, enfin des appartements, villas et terrains en Espagne, à Torremolinos et Marbella ${ }^{33}$.

Ajoutons enfin un troisième étage dans l'économie frontalière, maroco-espagnole du cannabis, celui des petits porteurs et des affaires ponctuelles, littéralement inquantifiable tant il est diffus. Ce sont des migrants originaires des régions productrices qui ramènent en Europe quelques dizaines de kilos à chaque vacances ; ce sont les chauffeurs routiers et chauffeurs de bus réguliers, qui, sûrs de quelques complicités policières gagnées dans la routine du passage ou dans les réseaux familiaux, passent eux aussi quelques kilos à chaque voyage ; ce sont les marins pêcheurs qui déchargent en mer à des bateaux espagnols, pêcheurs eux aussi de l'autre rive, quelques dizaines de kilos régulièrement; ce sont les marins des bateaux qui assurent les liaisons quotidiennes entre le Maroc, des ports de Tanger et Sebta, et le continent européen ; une économie diffuse, capillaire, mais qui reste cependant locale. Car il lui faut une totale impunité pour s'exercer et donc des complicités - parentales, villageoises, amicales.

Economie socialement diffuse à l'intérieur d'un territoire social transnational, telle apparaît l'économie locale du cannabis. Loin de ces systèmes mafieux que décrivent la plupart des chercheurs italiens, comme les travaux sur les cartels criminels en Amérique du Sud ${ }^{34}$.

33. La totalité de son patrimoine, en fait très classiquement placé aux noms de ses enfants, épouse et frères, est détaillé dans la Gazette du Maroc, n523, 5-11mai 2007.

34. Voir Cultures E Conflits, n³, 1991 (numéro spécial consacré aux trafics de drogue dans le monde et aux rapports aux politiques); Labrousse A., Colombie, conflit de la drogue ou conflit politico-militaire, CEMOTI, n³2, 2004. 
Plus globalement, on pourrait dire aussi que l'économie du cannabis prend place dans une « économie de bazar » généralisée, si l'on entend par là non pas une absence d'économie ${ }^{35}$, mais une forme économique basée sur le primat de l'échange sur tout autre relation économique (absence du salariat et de rapport d'exploitation), la réduction de l'échange à des interactions personnelles, «face à face », enfin des relations personnalisantes, de clientèle fidélisée. Ce sont là rassemblées les traits constitutifs de l'économie de bazar telle que la définit Clifford Geertz ${ }^{36}$ et qui me semble assez précisément ajustée à l'économie tangéroise en général telle que nous pouvons l'observer ${ }^{37}$. Est-ce suffisant pour expliquer l'absence radicale ici de toute violence « guerrière » et de toute organisation criminelle telles que décrites en Italie ou en Amérique du Sud ? Assurément non, mais les données manquent. Du moins faut-il faire l'hypothèse d'une confluence de phénomènes, qui sont, en vrac, l'affairisme et le mercantilisme comme «mentalité » et le bazar comme modèle culturel, le caractère diffus de l'économie qui fonctionne comme un système de régulation et de répartition inégale, (tout le monde touche un peu aux bénéfices des commerces), enfin la quasi totale absence de répression et de surveillance locale, du moins jusqu'à ces dernières années. Un faisceau de phénomènes combinés qui méritent d'être analysés dans leur conjonction pour expliquer la non-émergence sur le mode violent d'une classe moyenne affairiste ${ }^{38}$. Car pour autant, on assiste bien ici aussi à l'émergence sinon d'une «classe moyenne délinquante 39 » du moins d'une possibilité promotionnelle offerte à de nombreux acteurs par leur implication informelle dans les commerces transnationaux, criminels ou pas.

L'ensemble de l'affairisme tangérois met en lumière des réseaux d'acteurs très singuliers, au regard de ceux qui sont ailleurs impliqués dans les économies criminelles. L'expression « homme d'affaire » prête à ambiguiité. Car ici, on « est en affaire », bien plus que l'on en fait. Etre en affaire c'est «monter des coups », autre expression très usitée, c'est-à-dire investir un capital plus ou moins conséquent dans une opération, généralement commerciale, dont la légalité ne peut être très souvent que partielle puisqu'elle joue des différentiels frontaliers. Il peut être légal par exemple d'acheter sous douane 5000 chapeaux de paille fabriqués en Chine, comme il est légal, voire moral, d'en revendre une partie à des grossistes locaux. En revanche, un certain nombre de ces chapeaux, qui se vendent dans

35 . Ruggiero V., South N., « La ville de la fin de l'ère moderne en tant que bazar : marchés des stupéfiants, entreprise illégale et "barricades" ", Déviance et société, vol.20, n4, 1996.

36. Geertz C., "Suq: the bazaar economy in Sefrou", in Geertz C., Geertz H., Rosen L., Meaning and Order in Moroccan Society, Cambridge, Cambridge University Press, 1978.

37. Ajoutons, au regard de nos propres travaux sur le «commerce à la valise », que l'économie de bazar est aussi une manière de ramener les rapports d'échange à des logiques de «coups ", en métaphore au jeu. Voir Peraldi M, Cabas et containers, Paris, Maisonneuve et Larose, 2001.

38. Nous reprenons ici le propos de R. Catanzaro (Catanzaro R., Il Delitto come impresa. Storia sociale della mafia, op. cit.) qui suggère que la mafia peut être considérée comme un phénomène d'émergence d'une classe moyenne promotionnelle fondée sur l'extorsion et le prélèvement par la violence sur l'économie locale.

39. Ruggiero V., Delitti dei deboli e dei potenti, Milano, Bollati Boringhieri, 1999. 
les souks locaux, est sorti un beau soir, sous le regard aveugle d'un douanier à qui on a « acheté la route », jeté du haut d'une terrasse d'un entrepôt de la zone franche pour rejoindre, sans avoir payé les taxes, les étalages.

Ces hommes d'affaire peuvent investir aussi bien dans l'hôtellerie que dans le commerce, la contrebande, mettre une partie de leur capital dans des entreprises de transport, autre secteur industriel très florissant de la zone, participer au financement et au montage d'ateliers de confection, comme ils peuvent participer à des coups commerciaux sur de petites quantité de cannabis, leur rôle en la matière consistant à capitaliser l'achat du produit. Ils constituent moins qu'une classe sociale et plus que des individus dispersés, car ils se retrouvent en « cliques ", réunis par des sociabilités de circonstance, l'habitude de faire des affaires et une confiance pragmatique : on ne se fait confiance que d'une opération à l'autre sans "capitaliser », en quelque sorte, le crédit de confiance acquis dans l'opération. Seuls quelques hommes d'affaire installés depuis longtemps sur la place bénéficient d'une réputation qui les fait passer, à tort, même si elle les flatte, pour des «chefs ». La confiance, nous a dit l'un d'entre eux, c'est « jusqu'à la prochaine fois »... L'une de ces cliques sur laquelle s'est portée notre enquête est par exemple constituée de personnes de trente à cinquante ans, toutes diplômées, voire de formation universitaire ou technique. C'est bien en ce sens qu'elles constituent une classe moyenne. Le noyau central est constitué de personnes originaires de la même petite ville, auquel viennent s'ajouter des anciens amis qui ont effectué un séjour plus ou moins long en prison pour raisons politiques dans les « années de plomb » marocaines $40 \mathrm{et}$, plus épisodiquement, des personnes arrimées au groupe seulement par le même goût épicurien pour les lieux nocturnes, l'alcool (en pays musulman) et la fréquentation des prostituées. Autant de pratiques qui sont, en pays musulman, une manière de marquer une différence idéologique déviante et s'affirmer comme tel ${ }^{41}$. Ces trois cercles de sociabilité, «parrochial », affinitaire politique, sociabilités déviantes, impliquent un petit noyau de personnes en affaire qui constitue le cœur stable de la clique. Ils investissent dans des restaurants, des hôtels, des bars, à Tanger ou en Espagne. Ils sont marocains, hollandais, français, espagnols. Autour d'eux gravite une nébuleuse de relations plus ou moins régulièrement associées, la plupart du temps sur un mode festif, qui vont d'universitaires à des membres de partis et organisations politiques, magistrats, notables, élus locaux, entrepreneurs, artistes, etc., sans autre lien que la commune complicité des buveurs dans un monde où il est interdit de boire, et sans autre dépendance que des réseaux de dettes ponctuelles en permanence effacées puis réécrites.

Des liens faibles donc, selon la définition de M. Granovetter ${ }^{42}$, c'est-àdire des liens qui n'impliquent pas de dépendance statutaire ce qui, là encore,

40. Daoud Z., Maroc: les années de plomb, 1958-1988, chronique d'une résistance, Paris, Editions Manucius, 2007.

41 . Becker H., Outsiders, étude de sociologie de la déviance, Paris, Editions Métailié, 1985.

42. Granovetter M., "The strength of weak ties”, American Journal of Sociology, vol.78, n6, 1973. 
n'explique pas l'absence de violence mais entre dans le paysage social où se compose un système économique, criminel certes, du point de vue étroitement légal, mais encastré dans la société transnationale dont il est une pièce d'une autre manière ne le sont les organisations criminelles décrites par les chercheurs italiens.

Au point initial de cette recherche, nous conclurons par une hypothèse, susceptible de falsification et d'affinement: dans la logique des mondes urbains marocains dont nous analysons l'évolution, ce «moment criminel » dans le cycle capitaliste prend la forme sociale d'une double émergence : celle d'une part d'une classe moyenne urbaine, cosmopolite mais locale, impliquée dans une gamme large d'affaires latérales au cœur criminel des activités. Marginaux et «petits» dans les trafics, ces acteurs sont ainsi capables d'assurer la continuité de leur ancrage sociale et statutaire et, par là même, leurs capacités de promotion sociale, en s'écartant des activités ou des moments du cycle dans lesquels le recours à la violence est sinon nécessaire, du moins condition productive. Ce «monopole» de la violence est en revanche caractéristique de dispositifs et réseaux transnationaux de taille et de dimension capitalistes, notamment parce qu'ils poussent leurs investissements jusqu'au cœur de la machine capitaliste la plus vertueuse, dès l'instant qu'elle prend forme transnationale. Les cours de ces réseaux sont pour l'essentiel en Europe, à distance des lieux de production, mais très proches des centres d'affaires européens. 